\title{
Estudio urodinámico en la evaluación de la incontinencia urinaria femenina, con el nuevo sistema MoniTorr MR (urodinamia monocanal con medición de presión de retro-resistencia uretral). Experiencia con 100 pacientes
}

\author{
Solà Dalenz V, Ricci Arriola P, Pardo Schanz J. \\ Unidad de Uroginecología y Cirugía Vaginal. Departamento de Obstetricia y Ginecología. Clínica Las Condes.
} Santiago de Chile. Chile

Actas Urol Esp. 2008;32(3):325-331

ESTUDIO URODINÁMICO EN LA EVALUACIÓN DE LA INCONTINENCIA URINARIA FEMENINA, CON EL NUEVO SISTEMA MONITORR $^{\text {MR }}$ (URODINAMIA MONOCANAL CON MEDICIÓN DE PRESIÓN DE RETRO-RESISTENCIA URETRAL). EXPERIENCIA CON 100 PACIENTES

Objetivos: Correlacionar el diagnóstico clínico de la incontinencia urinaria con los resultados de la cistometría (LPP) y URP, obtenidas por medio de urodinamia monocanal. Evaluar la tolerancia de los pacientes a este examen.

Paciente y método: Entre agosto y diciembre del 2006, se realizaron 100 urodinamias monocanal con medición de URP, en las pacientes que consultaron por diagnóstico clínico de incontinencia de orina, en la Unidad de Uroginecología y Cirugía Vaginal, de Clínica Las Condes. Se correlacionó el diagnóstico clínico con los resultados de la urodinamia. Se aplicó una escala de tolerancia subjetiva del examen, donde 0 es la mayor tolerancia y 10 la menor.

Resultados: De las 66 pacientes con clínica de incontinencia de orina de esfuerzo: 10 fueron tipo 0, 2 tipo I, 23 tipo II, 1 tipo III, 17 tipo II+III, 1 detrusor hiperactivo, 5 tipo 0+detrusor hiperactivo, 3 tipo II+detrusor hiperactivo, 4 II+III+detrusor hiperactivo. De las 15 pacientes con IOM: 6 urodinamias fueron tipo 0, 2 tipo II, 2 tipo II+III, 3 detrusor hiperactivo, 1 tipo II+detrusor hiperactivo y 1 tipo III+detrusor hiperactivo. De las 16 pacientes con urgeincontinencia: 2 normal, 4 II+III, 7 detrusor hiperactivo, 1 II+detrusor hiperactivo y 2 II+III+detrusor hiperactivo. En 3 pacientes se solicitó una urodinamia como parte del estudio pre-operatorio, por corresponder respectivamente a dos casos de prolapso de cúpula y un cistocele, todos grado IV (POP-Q), con una gran potencialidad de incontinencia de orina después de la corrección. Presentaron respectivamente una incontinencia de esfuerzo tipo III+detrusor hiperactivo, una tipo II+III y una urodinamia normal. Todas las pacientes identificaron una tolerancia que clasificaron como 2 o 3 (escala de 0 a 10, donde 0 corresponde al grado mayor tolerancia y 10 al grado de menor tolerancia).

Conclusiones: La urodinamia monocanal más la medición de la URP es un examen complementario de gran utilidad en la objetivación y clasificación del diagnóstico de la incontinencia urinaria, sospechada por la clínica. Permite al médico planificar la solución adecuada para cada caso y tiene un alto grado de tolerancia en las pacientes.

Palabras clave: Urodinamia. Urodinamia Monocanal. URP. Urodinamia Multicanal. Incontinencia de Orina. Incontinencia de orina de esfuerzo.

\begin{abstract}
URODYNAMIC STUDY IN THE FEMALE URINARY INCONTINENCE EVUALUATION, WITH THE NEW MONITORR ${ }^{\text {MR }}$ SYSTEM (NONMULTICHANNEL URODYNAMIC WITH URETHRAL RETRO-RESISTANCE PRESSURE MEASURE). EXPERIENCE WITH 100 PATIENTS

Objective: To compare the clinical diagnosis of the urinary incontinence with the results of LPP and URP obtained by non-multichannel urodynamic. To evaluate the tolerance of the patients to this examination.

Pathient and Method: Between August and December 2006, 100 urodynamics were made in patients with clinical diagnosis of urinary incontinence, in the Urogynecology Unit of Clinica Las Condes. The clinical diagnosis was compared with the urodynamic results. A subjective tolerance scale was applied ( 0 was greater tolerance and 10 was maximum discomfort).

Results: In 66 patients with clinical diagnosis of IUS, the urodynamic registered 10 Type 0, 2 (I), 23 (II), 1 (III), 17 (II+III), 1 hyperactive detrusor, 5 ( $0+$ hyperactive detrusor), 3 (II+hyperactive detrusor) and 4 (II+III+hyperactive detrusor). In 15 with Mixed urinary incontinence, the urodynamic showed 6 (Type 0), 2 (II), 2 (II+III), 3 hyperactive detrusor, 1 (II+hyperactive detrusor) and 1 (III+hyperactive detrusor). In 16 patients with urgency incontinence, urodynamic showed 2 normal, 4 (II+III), 7 hyperactive detrusor, 1 (II+hyperactive detrusor) and 2 (II+III+hyperactive detrusor). In 3 patients (two vaginal cuff prolapse and one cistocele, degree IV POP-Q) the urodynamic was indicated in the pre-surgery study considering a great potentially incontinence after correction. Respectively, the patients presented: IUS type III+hyperactive detrusor, IUS type II+III and one normal. All patients expressed 2 and 3 degree tolerance (subjective scale: 1 major tolerance and 10 a minor tolerance).

Conclusions: The MoniTorr ${ }^{\mathrm{MR}}$ urodynamic is a complementary examination very useful in the study of the urinary incontinence. It allows planning the solution adapted for each case and has a high degree of tolerance in the patients.
\end{abstract}

Keywords: Urodinamyc. URP. Multichannel Urodynamic. Urinary Incontinence. Stress urinary incontinence. 
$\mathrm{L}_{\mathrm{p}}^{\mathrm{a}}$ a incontinencia de orina es una patología de alta prevalencia. Se estima que en Europa el 55\% de las mujeres son portadora de esta condición $^{1,2}$, impactando negativamente la calidad de vida. La anamnesis y la historia clínica no siempre son suficientes para asegurar un correcto diagnóstico frente a una incontinencia urinaria ${ }^{3}$. Los sintomas que nos relata la paciente, no siempre reflejan en forma precisa el tipo de incontinencia que tienen ${ }^{4}$. Además los tratamientos tanto médicos como quirúrgicos han aumentado tanto en número como en sofisticación ${ }^{5}$. Por este motivo, la urodinamia, se constituye como una herramienta que nos permite objetivar y clasificar la incontinencia de orina percibida por la paciente. De esta forma, se puede planificar la mejor solución en el tratamiento de la incontinencia para cada paciente ${ }^{6}$. La literatura científica ha demostrado el rol positivo de la urodinamia, en la prevención de la falla y las complicaciones del tratamiento quirúrgico $^{7-16}$. Por otro lado, la alta prevalencia de esta condición patológica, necesariamente nos lleva a tener en cuenta, los altos costos tanto individuales como para los servicios de salud, al implementar técnicas de estudio como tratamientos específicos para dar una buena solución ${ }^{17}$. Es así como aparece recientemente, la urodinamia monocanal con medición de presión de retro-resistencia uretral. Una técnica más fácil de aplicar y de menor costo tanto de implementación como por examen para la paciente, si la comparamos con la urodinamia multicanal.

Con el objetivo de correlacionar el diagnóstico clínico de pacientes con incontinencia urinaria, con los resultados de la urodinamia monocanal (MoniTorr ${ }^{\mathrm{MR}}$, Gynecare, Johnson \& Johnson) ${ }^{18,19}$ (Fig. 1), y determinar la tolerancia frente a este examen, presentamos nuestra experiencia en el estudio de 100 pacientes ingresadas a la Unidad de Uroginecología de Clínica Las Condes.

\section{MATERIAL Y MÉTODOS}

Entre agosto y diciembre del 2006 se realizaron 100 urodinamias monocanal con medición de presión de retro-resistencia uretral (URP en inglés) ${ }^{18,19}$, en pacientes derivadas a la Unidad de Uroginecología de Clínica Las Condes, con el diagnóstico clínico de incontinencia de orina de esfuerzos, urgeincontinencia, mixta, potencial o diagnóstico dudoso.

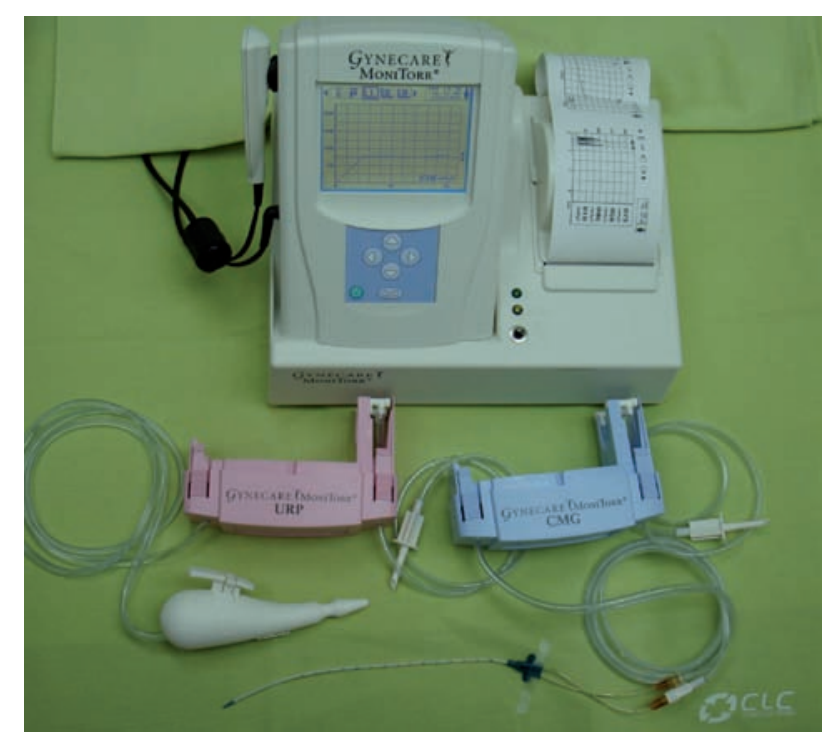

FIGURA 1. MoniTorr (Gynecare, Johnson \& Johnson) para urodinamia. Se presentan ambos dispositivos (CMG y URP) y el sistema de impresión de registro gráfico.

Se utilizó el sistema MoniTorr ${ }^{\mathrm{MR}}$ (Gynecare, Worldwide, división de Ethicon INC, compañía de Johnson \& Johnson, Somerville, New Jersey), que está compuesto por un microprocesador (unidad electrónica portátil y recargable) y dos dispositivos desechables (Fig. 2). Uno es para medir el URP y el otro corresponde al CMG (cistometrograma). La unidad electrónica se conecta a cada "cartridge" o cartucho desechables, según la medición a realizar. Estos funcionan en combinación con el hardware y software específico de la unidad utilizada. La unidad electrónica posee un visor y un control remoto. El cartucho de CMG posee un catéter de 7 french, que

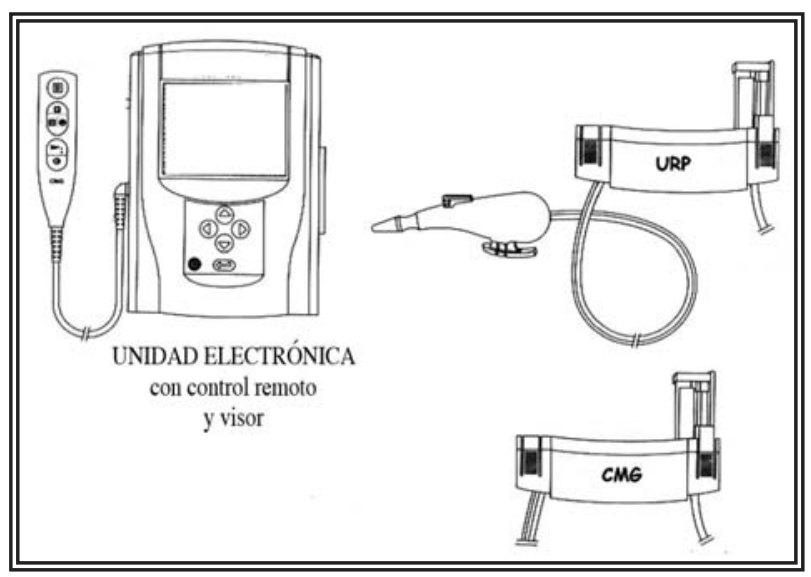

FIGURA 2. Esquema de los componentes del sistema MoniTorrMR. Unidad electrónica, dispositivo URP desechable, dispositivo CMG desechable. 
se introduce en la uretra para realizar la medición, en cambio el de URP posee un sistema provisto de un cono que se coloca como tapón en la uretra (Figs. 3, 4 y 5).

A todas las pacientes se les solicitó este examen como parte del estudio de objetivación y clasificación de una incontinencia de orina, o en la potencialidad de su aparición, diagnosticada previamente por la clínica (anamnesis y examen físico).

Para clasificar el grado de incontinencia objetivado por la urodinamia monocanal con medición de URP, se utilizó la clasificación de McGuire et al. ${ }^{20}$ (Tabla 1).

\section{Criterio de exclusión}

1. Infección del tracto urinario. En todas las pacientes se confirmó la inexistencia de una infección urinaria o una bacteriuria asintomática, por medio de un sedimento de orina y urocultivo.

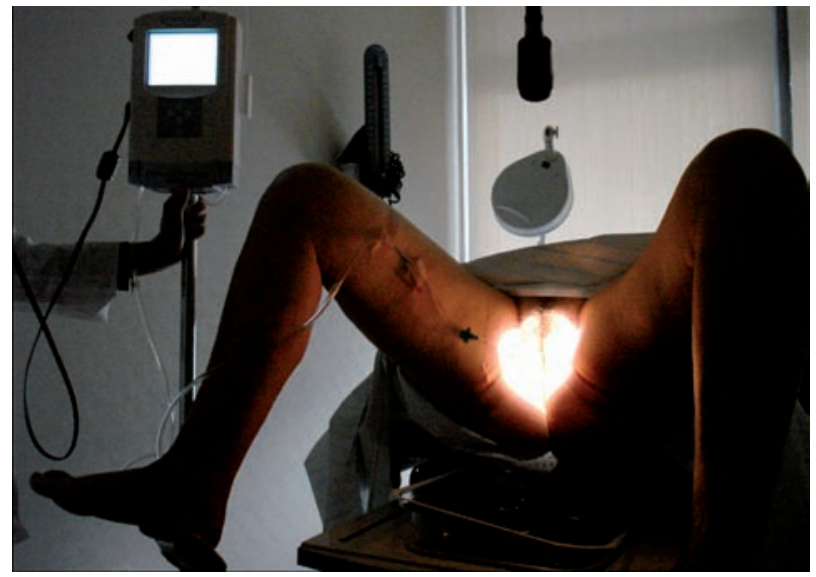

FIGURA 3. Paciente durante examen de urodinamia monocanal. Se aprecia dispositivo de CMG en utilización.

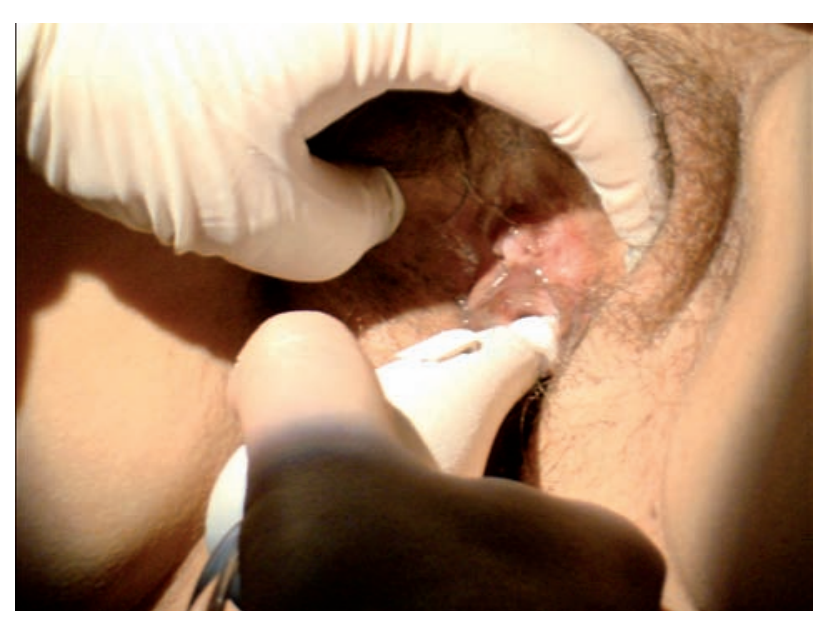

FIGURA 4. Introducción del cono de URP en la entrada uretral.

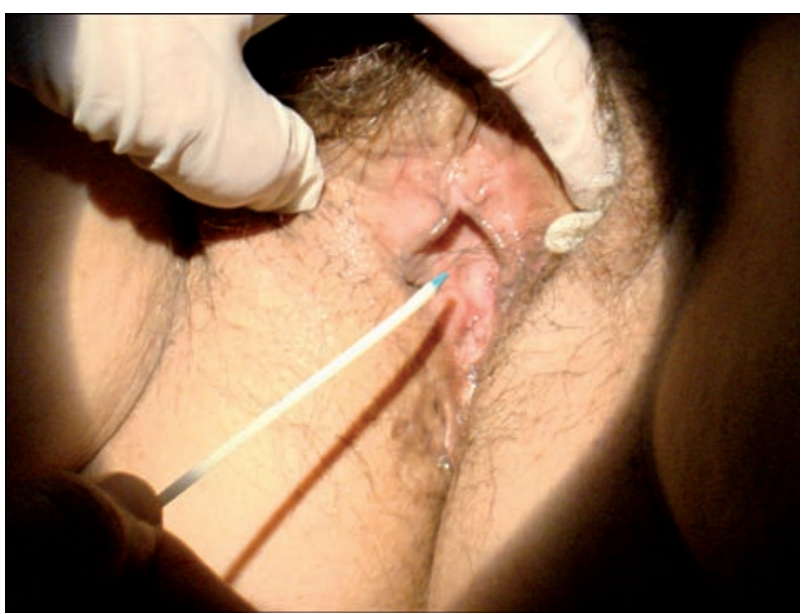

FIGURA 5. Introducción en uretra del catéter de CMG (Catéter de 7 french).

Tabla 1. Clasificación de la incontinencia de orina de esfuerzo según estudio urodinámico.

\begin{tabular}{ll}
\hline Tipo & Caracteristica \\
\hline Tipo 0: & $\begin{array}{l}\text { Incontinencia referida por la paciente, pero } \\
\text { que no es reproducida por el examen clínico o } \\
\text { por el estudio urodinámico }\end{array}$ \\
Tipo I: & $\begin{array}{l}\text { Incontinencia por esfuerzo con presión de } \\
\text { pérdida sobre } 90 \mathrm{cmH}_{2} \mathrm{O} \text { e hipermovilidad de } \\
\text { cuello y uretra menor a } 2 \mathrm{~cm}\end{array}$ \\
Tipo II: & $\begin{array}{l}\text { Incontinencia por esfuerzo con presión de } \\
\text { pérdida sobre } 90 \mathrm{cmH}_{2} \mathrm{O} \text { e hipermovilidad de }\end{array}$ \\
& $\begin{array}{l}\text { cuello y uretra mayor a } 2 \mathrm{~cm} \\
\text { Tipo III: }\end{array}$ \\
& $\begin{array}{l}\text { Insuficiencia uretral intrínseca, con presión } \\
\text { de pérdida inferior a } 60 \mathrm{cmH}_{2} \mathrm{O}\end{array}$
\end{tabular}

Clasificación de McGuire et al. ${ }^{20}$

2. Obstrucción uretral. Casos en que el estudio debe realizarse con urodinamia multicanal. Poco frecuente en mujeres, un ejemplo de estos casos es el prostatismo en los varones.

El grupo en estudio presentó una mediana de edad de 52 años, IMC de 26, paridad de 3. Presentaban antecedente de histerectomía 20 pacientes. Diez registraban alguna cirugía anti-incontinencia (Tabla 2).

En los casos que presentaban prolapso, se redujo con una valva de Breisky antes de realizar la urodinamia, para no alterar los resultados.

Antes de la urodinamia, se realizó a todas las pacientes un Q-Tip-Test como parte del estudio, para registrar la movilidad uretral.

Se registró el diagnóstico clínico de la incontinencia de orina por la cual se indicó el examen, y se comparó con el resultados obtenidos en la urodinamia monocanal con medición de URP. 
Tabla 2. Características de las pacientes en estudio con urodinamia monocanal con medición de URP

\begin{tabular}{|c|c|}
\hline Característica & Número \\
\hline Edad & 52 años (mediana) \\
\hline Paridad & 3 (mediana) \\
\hline IMC & 26 (mediana) \\
\hline Antecedente Histerectomía & 20 \\
\hline $\begin{array}{l}\text { Antecedente de cirugía } \\
\text { anti-incontinencia } \\
\text { Puntos de Kelly } \\
\text { Operación de Burch } \\
\text { Sling suburetral }\end{array}$ & $\begin{array}{l}10 \\
1 \text { (con histerectomía) } \\
6 \text { (4 con histerectomia) } \\
3 \text { (2 TVT, } 1 \text { TOT })\end{array}$ \\
\hline $\begin{array}{l}\text { Tolerancia al examen } \\
\text { (escala } 0 \text { al } 10 \text { ) } \\
\qquad 2-3\end{array}$ & Todas (100 pacientes) \\
\hline
\end{tabular}

Total 100 pacientes

Una vez concluida la urodinamia, se solicitó a cada paciente, que clasificara el grado de tolerancia frente al examen. Para ello se utilizó una escala subjetiva de 0 a 10, donde el número cero correspondía al grado de mayor tolerancia y el diez a la mínima tolerancia.
En 15 pacientes que habían sido sometidas en algún momento del estudio de su incontinencia de orina, a una urodinamia multicanal, se les solicitó que registraran el grado de tolerancia con la misma escala en esa ocasión.

\section{RESULTADOS}

De las 100 pacientes a las cuales se solicitó la urodinamia por clínica (anamnesis y examen físico), el $66 \%$ fue por incontinencia de esfuerzo, 15\% urgencia, $16 \%$ mixta y $3 \%$ por incontinencia potencial (Fig. 6).

La urodinamia monocanal registró un 3\% de exámenes dentro de límites normales, 68\% de IOE, 18\% IOM y 11\% IOU (Tabla 3).

Al correlacionar los diagnósticos clínicos que motivaron la indicación del estudio urodinámico, de las 66 pacientes con clínica de incontinencia de orina de esfuerzo: 10 fueron tipo 0, 2 tipo I, 23 tipo II, 1 tipo III, 17 tipo II+III, 1 detrusor hiperactivo, 5 tipo 0+detrusor hiperactivo, 3 tipo II+detrusor hiperactivo, 4 II+III+detrusor hiperactivo (Tabla 4).

Tabla 3. Relación del diagnóstico clínico (anamnesis y examen físico) de la incontinencia de orina versus el resultado de la urodinamia monocanal con medición de URP

\begin{tabular}{lccccc}
\hline $\begin{array}{l}\text { Clínica } \\
\text { Característica }\end{array}$ & NORMAL & IOE & $\begin{array}{c}\text { Hallazgo en urodinamia } \\
\text { IOM }\end{array}$ & IOU & Total \\
\hline IOE & - & 53 & 12 & 1 & 66 \\
IOM & - & 10 & 2 & 3 & 15 \\
IOU & 2 & 4 & 3 & 7 & 16 \\
IP & 1 & 1 & 1 & 0 & 3 \\
Total & 3 & 68 & 18 & 11 & 100 \\
\hline
\end{tabular}

IOE (Incontinencia de orina de esfuerzo). IOM (Incontinencia de orina mixta) IOU (Incontinencia de orina de urgencia. IP (Incontinencia potencial)

Tabla 4. Relación del diagnóstico clínico (anamnesis y examen físico) de la incontinencia de orina versus el resultado de la urodinamia monocanal con medición de URP. Se detalla el tipo de IOE y sus asociaciones.

\begin{tabular}{|c|c|c|c|c|c|c|c|c|c|c|c|c|}
\hline \multirow{2}{*}{$\begin{array}{l}\text { Clinica } \\
\text { Caracteristica }\end{array}$} & \multirow[b]{2}{*}{ Normal } & \multirow[b]{2}{*}{$\mathbf{0}$} & \multirow[b]{2}{*}{$\mathbf{I}$} & \multirow[b]{2}{*}{ II } & \multirow[b]{2}{*}{ III } & \multicolumn{4}{|c|}{ Hallazgo en urodinamia } & \multirow[b]{2}{*}{$\mathbf{I I I + D H}$} & \multirow[b]{2}{*}{$\mathbf{I I}+\mathbf{I I I}+\mathbf{D H}$} & \multirow[b]{2}{*}{ Total } \\
\hline & & & & & & II+III & DH & O+DH & II+DH & & & \\
\hline IOE & $\longrightarrow$ & 10 & 2 & 23 & 1 & 17 & 1 & 5 & 3 & - & 4 & 66 \\
\hline IOM & $\longrightarrow$ & 6 & - & 2 & - & 2 & 3 & - & 1 & 1 & - & 15 \\
\hline IOU & 2 & - & - & - & - & 4 & 7 & $\longrightarrow$ & 1 & - & 2 & 16 \\
\hline Potencial & 1 & 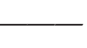 & - & - & - & 1 & - & - & - & 1 & - & 3 \\
\hline Total & $3 \%$ & $16 \%$ & $2 \%$ & $25 \%$ & $1 \%$ & $24 \%$ & $11 \%$ & $5 \%$ & $5 \%$ & $2 \%$ & $6 \%$ & 100 \\
\hline
\end{tabular}

IOE (Incontinencia de orina de esfuerzo). IOM (Incontinencia de orina mixta)

IOU (Incontinencia de orina de urgencia). IP (Incontinencia potencial)

DH (Detrusor hiperactivo) 


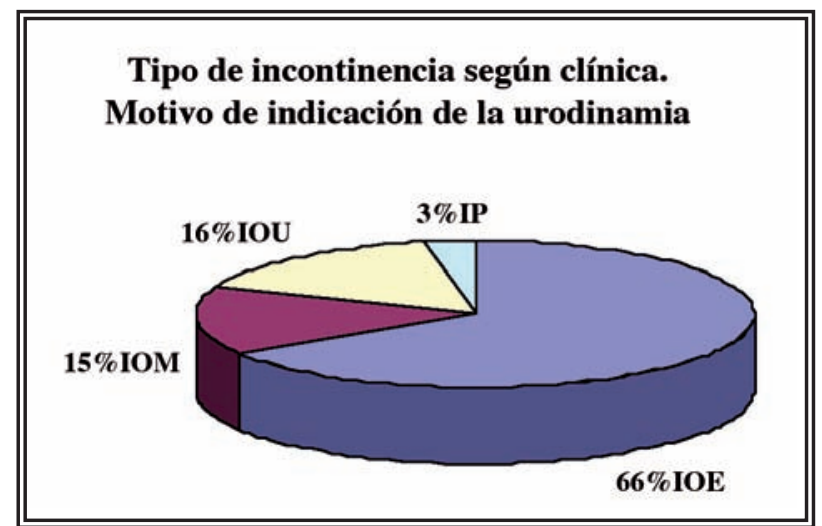

FIGURA 6. Tipo de Incontinencia de Orina según clínica en pacientes derivadas a estudio urodinámico.

De las 15 pacientes con IOM: 6 urodinamias fueron tipo 0, 2 tipo II, 2 tipo II+III, 3 detrusor hiperactivo, 1 tipo II+detrusor hiperactivo y 1 tipo III+detrusor hiperactivo (Tabla 4).

De las 16 pacientes con urgeincontinencia: 2 normal, 4 II+III, 7 detrusor hiperactivo, 1 II+detrusor hiperactivo y 2 II+III+detrusor hiperactivo (Tabla 4).

En 3 pacientes se solicitó una urodinamia como parte del estudio pre-operatorio, por corresponder respectivamente a dos casos de prolapso de cúpula y un cistocele, todos grado IV (POP-Q), con una gran potencialidad de incontinencia de orina después de la corrección. Los casos de prolapso de cúpula presentaron respectivamente una urodinamia con incontinencia de esfuerzo tipo III+detrusor hiperactivo y el otro una tipo II+III. El caso de cistocele presentó una urodinamia normal (Tabla 4).

Respecto a la tolerancia del examen, todas identificaron los grados 2 o 3 (escala de 0 a 10 , donde 0 corresponde al grado mayor tolerancia y 10 al grado menor de tolerancia).

De las 15 (15\%) pacientes que registraban entre sus antecedentes, algún estudio de urodinamia multicanal en algún momento de su vida. Se les pidió que clasificaran su tolerancia en esa ocasión en la misma escala. Todas la clasificaron entre 7 y 8 .

De las 89 pacientes que presentaban IOE urodinámica, 3 de ellas tenían DIE pura, 56 presentaban hipermovilidad uretral sin daño esfinteriano y 30 con ambas condiciones (Fig. 7).

\section{DISCUSIÓN}

Cuando se implementa una nueva técnica diagnóstica, es muy importante identificar los beneficios que nos puede aportar en la investigación e identifi- cación de una patología. Una buena historia y los parámetros clínicos no siempre aseguran la sensibilidad de esos síntomas, por lo que la urodinamia entrega una herramienta objetiva, fundamental para planificar la terapia más adecuada ${ }^{7-10}$.

Weidner et al. ${ }^{21}$ en un estudio de 950 pacientes con clínica de incontinencia de orina, concluye que los sintomas no deben tomarse como base para planificar un tratamiento quirúrgico. Cundiff et al. ${ }^{22}$ en otro estudio de 535 pacientes concluye que los síntomas permiten identificar menos de un cuarto de las incontinencias por estrés puras o detrusor inestable.

Actualmente y en base a los diferentes estudios publicados, no debiéramos discutir los beneficios de realizar una urodinamia para objetivar y clasificar una incontinencia de orina, lo que ha sido demostrado con la urodinamia multicanal. Sin embargo, los altos costos de esta tecnología, necesariamente se reflejarán en un mayor costo por examen, lo que sumado a la alta prevalencia de la incontinencia de orina, llevan a grandes gastos que deben asumir los pacientes y/o los sistemas de salud. Por otro lado, el número de pacientes con incontinencia de orina, necesariamente aumentará, ya que la sobrevida de la mujer también lo ha hecho. Además, cada día la mujer nos consultará en mayor proporción por una solución adecuada a esta patología, para permitirse una mejor calidad de vida durante los años venideros. Actualmente se estima que una de cada 4 pacientes busca ayuda por este problema ${ }^{23}$. En aquellas pacientes en que se estudia la incontinencia, surge esta nueva tecnología, de urodinamia monocanal, como una alternativa eficaz y viable frente a la urodinamia multicanal. Con una implementación de menor costo (10 veces menor que la tecnología multicanal), que permite abaratar los precios por examen (30\% menos). Se trata de un equipo más económico, amigable, portátil y pequeño.

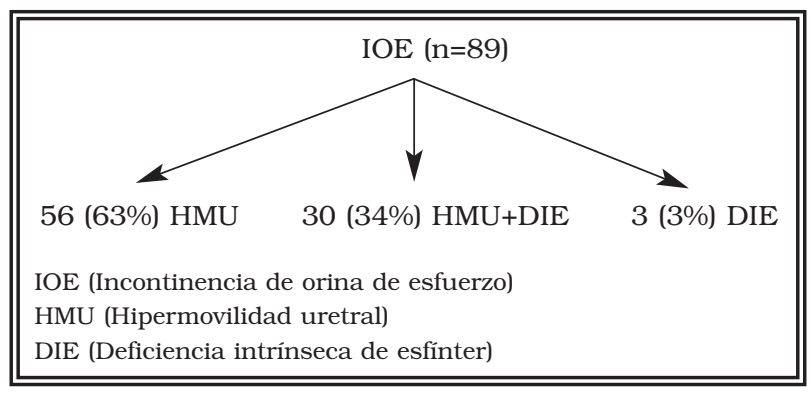

FIGURA 7 
Se trata de una tecnología más sencilla y menos invasiva, y por ello mejor tolerada por la paciente. Que permite estudiar la incontinencia urinaria en mujeres, para objetivar y clasificar el tipo de incontinencia, y así planificar una solución adecuada para cada paciente. La simplificación de cualquier examen que se realice en los genitales femeninos, permite muchas veces reducir el malestar y la vergüenza, que presentan estas mujeres sometidas a estas pruebas diagnósticas. Sin embargo, su principal contraindicación es aquella paciente que presenta alguna patología obstructiva, en la cual ante su necesidad, se debe indicar un estudio multicanal.

Entre las indicaciones de un estudio urodinámico, también consideramos aquellos casos en que la anatomía vaginal se encuentra muy alterada, como lo puede ser un caso de cistocele o de prolapso de cúpula vaginal severos ${ }^{24}$. Muchas veces al corregir estos grandes prolapsos genitales, aparece posteriormente una incontinencia oculta, que no se expresaba debido a la distorsión anatómica del complejo vesicouretral. Un estudio pre-operatorio adecuado, con reducción completa del prolapso ${ }^{25}$, nos permitirá predecir la necesidad de una cirugía antiincontinencia, en forma concomitante. Durante el 2000, Klutke y Ramos, publicaron su experiencia, demostrando la potencial aparición de incontinencia, utilizando pesarios antes de la cirugía ${ }^{26}$

Respecto a la asociación de incontinencia de orina tipo II y III, creemos que cada día sería más factible encontrar. Entre los dos extremos, es decir tipo II o III puros, existiría una gran gama de pacientes con un mayor o menor componente de cada una de éstas. Esto queda reflejado en nuestros resultados y constituye uno de los temas de estudio actuales de nuestro grupo de trabajo.

Actualmente, las pacientes con IOE asociadas a hipermovilidad uretral sin daño esfinteriano intrínseco (IOE tipo 0, I y II), en caso de planificar una cirugía, son tratadas en nuestra Unidad con cinta transobturatriz (TVT-O) como primera opción. En cambio, las que tienen hipermovilidad con daño intrínseco, son tratadas con TVT retropúbico, registrando una experiencia de 15 casos, con un 100\% de curación, con un seguimiento máximo de 32 meses (pacientes en seguimiento actualmente). La efectividad del TVT en la incontinencia de orina de esfuerzo es conocida, pero también se han publica- do buenos resultados en casos de incontinencia $\operatorname{mixta}^{27}$ y en pacientes con deficiencia intrínseca de esfinter con hipermovilidad uretral ${ }^{28}$.

Kayigil et al. ${ }^{29}$ durante 1999, mostraron que en 50 mujeres con IOE, se presentó un $72 \%$ de hipermovilidad uretral y un $26 \%$ de hipermovilidad asociada a deficiencia intrínseca de esfinter. En la presente experiencia, de las 89 pacientes con IOE en la urodinamia, 63\% tenían hipermovilidad uretral, $34 \%$ hipermovilidad asociada a deficiencia intrínseca de esfínter y un 3\% de deficiencia intrínseca de esfínter pura. Kayigil concluye que el alto número de deficiencias intrínsecas de esfínter en pacientes con hipermovilidad uretral indican que la incidencia de incontinencia de esfuerzo puede ser mayor a lo que se creía previamente, y esto puede constituir la explicación de los casos de falla después de suspensión del cuello vesical ${ }^{29}$

Por otro lado, esta técnica de urodinamia permite ofrecer un estudio que es muy bien tolerado, tal como fue registrado por nuestras pacientes. Pero esto no nos debe hacer olvidar, que al igual que la urodinamia multicanal, debe realizarse con una buena técnica aséptica, o de lo contrario podríamos introducir bacterias al sistema urinario. En estudios con urodinamia multicanal, aunque escasos, se han registrado casos de bacteriemia posteriormente al examen $^{30}$, lo que teóricamente si no tomamos las precauciones, también se podría producir con la monocanal.

\section{CONCLUSIONES}

La urodinamia monocanal con medición de URP es un método que nos permite objetivar y clasificar el tipo de incontinencia de orina sospechado por la clínica (anamnesis y examen físico). Se trata de un método que entrega las herramientas para planificar la solución más adecuada, según las características de cada paciente. Según nuestra experiencia, se trata de un método que posee un alto grado de tolerancia por las pacientes que lo requieren.

\section{REFERENCIAS}

1. Thom D. Variation in estimates of urinary incontinence prevalence in the community: effects of differences in definition, population characteristics and study type. J Am Geriatr Soc. 1998;46(4):473-480.

2. Stuck AE, Elkuch P, Dapp U, Anders J, Iliffe S, Swift CG. Feasibility and yield of a self-administered questionnaire for health risk appraisal in older people in three European countries. Age Ageing. 2002;31(6):463-467. 
3. Cardozo LD, Stanton SL. Genuine stress incontinence and detrusor instability-a review of 200 patients. Br J Obstet Gynaecol. 1980;87(3):184-190.

4. Jarvis GJ, Hall S, Stamp S, Millar DR, Johnson A. An assessment of urodynamic examination in incontinent women. $\mathrm{Br} \mathrm{J}$ Obstet Gynaecol. 1980;87(10):893-896.

5. Takacs EB, Zimmern PE. Recommendations for urodynamic assessment in the evaluation of women with stress urinary incontinence. Nat Clin Pract Urol. 2006;3(10):544-550.

6. Thompson PK, Duff DS, Thayer PS. Stress Incontinence in Women Under 50: Does Urodynamics Surgical Outcomes?. Int Urogynecol J Pelvic Floor Dysfunct. 2000;11(5):285-289.

7. Summitt RL, Stovall TG, Bent AE, Ostergard DR. Urinary incontinence: correlation of history and brief office evaluation with multichannel urodynamic testing. Am J Obstet Gynecol. 1992;166(6 Pt 1):1835-1844; discussion 1840-1844.

8. Sand PK, Hill RC, Ostergard DR. Incontinence history as a predictor of detrusor stability. Obstet Gynecol. 1988;71(2):257260 .

9. Korda A, Krieger M, Hunter P, Parkin G. The value of clinical symptoms in the diagnosis of urinary incontinence in the female. Aust NZ Obstet Gynaecol. 1987;27(2):149-151.

10. Ng RK, Murray A. Can we afford to take short cuts in the management of stress urinary incontinence?. Singapore Med J. 1993;34(2):121-124.

11. Dwyer PL, Desmedt E. Impaired bladder emptying in women. Aust N Z J Obstet Gynaecol. 1994;34(1):73-78.

12. Stanton SL, Ozsoy C, Hinton P. Voiding difficulties in the female: prevalence, clinical and urodynamic review. Obstet Gynecol. 1983;61(2):144-147.

13. Khan Z, Mieza M, Bhola A. Relative usefulness of physical examination, urodynamic and roentgenography in the diagnosis of urinary stress incontinence. Surg Gynecol Obstet. 1988;167(1): 39-44.

14. Byrne DJ, Stewart PA, Gray BK. The role of urodynamics in female urinary stress incontinence. Br J Urol. 1987;59(3):228229.

15. Powell PH, Shepherd AM, Lewis P, Feneley RC. The accuracy of clinical diagnoses assessed urodynamically. Prog Clin Rs. 1981; 78:201-203

16. Everaert K, Van Laecke E, De Muynck M, Peeters H, Hoebeke P. Urodynamics assessment of voiding dysfunction and dysfunctional voiding in girls and women. Int Urogynecol $\mathrm{J}$ Pelvic Floor Dysfunct. 2000;11(4):254-264.

17. Martin JL, Williams KS, Abrams KR, Turner DA, Sutton AJ, Chapple C, et al. Systematic review and evaluation of methods of assessing urinary incontinence. Health Technol Assess. 2006;10(6): 1-132, III-IV.

18. Slack M, Culligan P, Tracey M, Hunsicker K, Patel B, Sumeray M. Relationship of urethral retro-resistance pressure to urodynamic measurements and incontinence severity. Neurourol Urodyn. 2004;23(2):109-114.
19. Slack M, Tracey M, Hunsicker K, Godwin A, Patel B, Sumeray M. Urethral retro-resisteance pressure: a new clinical measure of urethral function. Neurol Urodyn. 2004;23(7):656-661.

20. McGuire EJ, Fitzpatrick CC, Wan J, Bloom D, Sanvordenker J, Ritchey M, et al. Clinical assessment of urthral sphincter function. J Urol. 1993;150(5 Pt 1):1452-1454.

21. Weidner AC, Myers ER, Visco AG, Cundiff GW, Bump RC. Which women with stress incontinence require urodynamic evaluation?. Am J Obstet Gynecol. 2001;184(2):20-27.

22. Cundiff GW, Harris RL, Coates KW, Bump RC. Clinical predictors of urinary incontinence in women. Am J Obstet Gynecol. 1997;177(2):262-266; discussion 266-267.

23. Nygaard IE, Heit M. Stress urinary incontinence. Obstet Gynecol. 2004;104(3):607-620

24. Bergman A, Koonings PP, Ballard CA. Predicting postoperative urinary incontinence development in women undergoing operation for genitourinary prolapse. Am J Obstet Gynecol. 1988; 158(5):1171-1175.

25. Rosenzweig BA, Pushkin S, Blumenfeld D, Bhatia NN. Prevalence of abnormal urodynemic test in continent women with severe genitourinary prolapse. Obstet Gynecol. 1992;79(4): 539-542.

26. Klutke JJ, Ramos S. Urodynemic outcome alter for severe prolapse and potencial stree incontinence. Am J Obstet Gynecol. 2000;182(6):1378-1381.

27. Rezapour M, Ulmsten U. Tension-Free vaginal tape (TVT) in women with mixed urinary incontinence-a long term follow-up. Int Urogynecol J Pelvic Floor Dysfunct. 2001;12 Suppl 2:S1518.

28. Rezapour M, Ulmsten U. Tension-free vaginal tape (TVT) in stress incontinent women with intrinsic sphincter deficiency (ISD)-a long term follow-up. Int Urogynecol $\mathrm{J}$ Pelvic Floor Dysfunct. 2001;12 Suppl 2:S12-14.

29. Kayigil O, Iftekhar Ahmed S, Metin A. The coexistence of intrinsic sphincter deficiency with type II stress incontinence. J Urol. 1999;162(4): 1365-1366.

30. Onur R, Orden M, Orhan I, Kalkan A, Semercioz A. Incidente of bacteraemia after urodynamic study. J Hosp. Infect. 2004;57 (3):241-244

Correspondencia autor: P. Ricci Arriola

Unidad de Uroginecología y Cirugía vaginal

Departamento de Obstetricia y Ginecología.

Clínica Las Condes

Lo Fontecilla 441, Las Condes. Santiago de Chile. Chile

Tel.: (56-2) 2104000 (56-2) 2104610

E-mail autor: pricci@clinicalascondes.cl, pricci@vtr.net

Información artículo: Original - Incontinencia urinaria femenina

Trabajo recibido: enero 2007

Trabajo aceptado: noviembre 2007 